\title{
Intense Use of Fracture-Associated Drugs Among Medicare Beneficiaries in Long-term Care
}

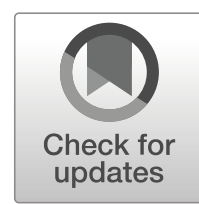

$\mathrm{J}$ Gen Intern Med 36(6):1818-20

DOI: $10.1007 / \mathrm{s} 11606-020-05859-2$

(c) Society of General Internal Medicine 2020

\section{INTRODUCTION}

Among older adults, common efforts to assess polypharmacy (multiple medications) often focus on simple drug count. Our past work exploring receipt of fractureassociated drugs (FADs) and fractures suggests specific drug combinations may matter more than absolute drug count. We found, in the general Medicare population, hip fracture risk rises steeply as people receive increasing numbers of concurrent FADs, yet this risk decreases slightly with higher intensity of non-FADs. ${ }^{1}$ To extend this previous work, we measure FAD and concurrent FAD receipt for a population particularly vulnerable to adverse drug effects - elderly living in long-term care (LTC). ${ }^{2} \mathrm{We}$ quantify FAD exposure in this population and compare it with the community-dwelling population to begin exploring an important potentially avoidable risk.

\section{METHODS}

Data Sources and Cohort. We used Medicare parts A (inpatient) and B (outpatient) administrative data, 2004-2014, and Medicare part D (prescription) records, 2006-2014, for a 40\% random sample of fully enrolled, fee-for-service (FFS) beneficiaries as previously described. ${ }^{1}$ From the overall sample, we identified a subcohort with prescription fill records suggestive of longterm care or assisted-living settings, namely, $50 \%$ or more of annual prescription fills dispensed by a LTC pharmacy. $^{3}$

Prescription Drug Receipt. We identified 21 distinct FAD groups including antidepressants, antipsychotics, loop diuretics, proton pump inhibitors (PPIs), and opioids. ${ }^{1} \mathrm{We}$ used fill records to identify medications received and "days supply" to set duration. For each person day, the status for each specific drug group of interest was assigned as exposed or not exposed.

Received March 23, 2020

Accepted April 13, 2020

Published online June 4, 2020
Statistical Analysis. Frequency distributions of FAD receipt $(1,2$, and 3 or more) and specific FAD combinations were determined and described as \% of total person years (PY) observed for LTC and community-dwelling Medicare beneficiaries. FAD Receipt patterns were compared with the chisquare test using SAS software, version 9.4. Significant associations were considered at $p$ value $<0.05$.

\section{RESULTS}

From the sample $(5,292,510$ beneficiaries, $22,573,534$ person years (PY)), 422,111 beneficiaries and $1,803,544$ PY met LTC cohort criteria. Overall and in the LTC cohort, $61.1 \%$ and $71.4 \%$ of beneficiaries were females; $8.3 \%$ and $11.9 \%$ were black; the mean age was 77.2 years (standard deviation (SD) 7.3) and 84.0 years (SD 8.3), respectively. Beneficiaries in the LTC cohort were more likely than the community-dwelling, elderly population to receive part D low-income subsidy $(73.6 \%$ versus $27.5 \%, p<0.001)$ and to be Medicaid-eligible $(68.2 \%$ versus $23.1 \% p<0.001)$.

Compared with the community-dwelling Medicare population, LTC beneficiaries received more FADs alone and in combination (Fig. 1). Among LTC beneficiaries, $15 \%$ of PY were characterized by two, and $19 \%$ by three-or-more concurrent FADs; these measures in the total population were $12 \%$ and $8 \%$, respectively ( $p<0.001$ for both). Specific, common, concurrent twoway FAD combinations in the LTC cohort included PPI plus selective serotonin reuptake inhibitors (SSRI), SSRI plus second-generation antipsychotics (SGAP), SSRI plus loop diuretics, PPI plus loop diuretics, and SSRI plus opioids. These combinations were received less commonly in the community-dwelling population (Table 1).

\section{DISCUSSION}

Medicare LTC beneficiaries received concurrent FADs more often than their non-LTC peers, raising concern for potential iatrogenic and possibly avoidable fracture risk. ${ }^{1}$ This higher intensity of prescription drug receipt included co-exposures to psychotropics and opioids and potentially discretionary (e.g., PPI) or relatively contraindicated medications (e.g., SGAP). ${ }^{4}$ 


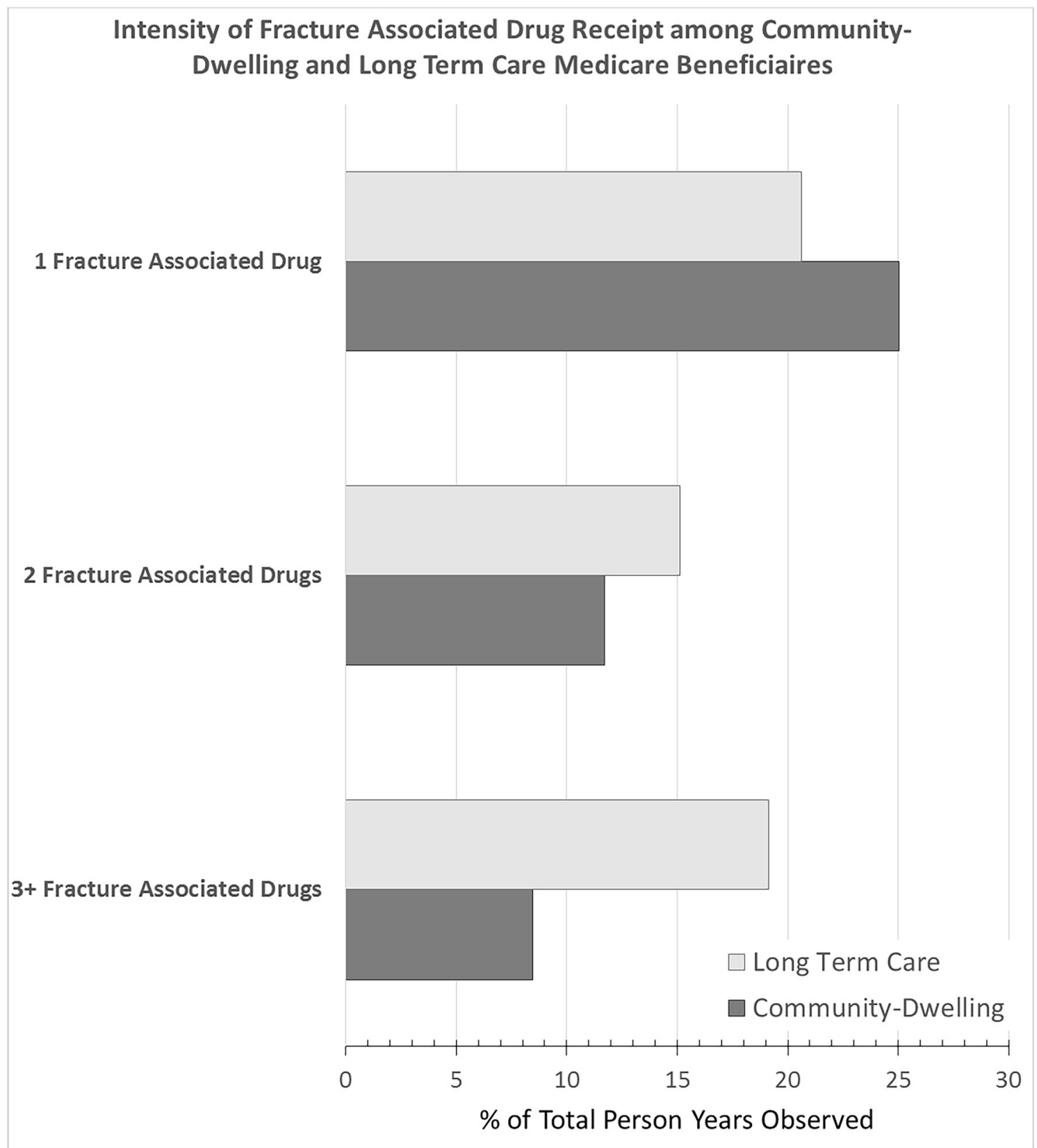

Fig. 1 Receipt of fracture-associated drugs (FADs) as \% total person years observed in a $40 \%$ random Medicare sample and the long-term care sub-cohort. For FADs, receipt status for 21 single drugs and all two-way concurrent combinations is classified for each person, for each day of observation. 3+ fracture-associated drugs is a classification of person days with 3 or more concurrent FADs. Long-term care individuals are those with $50 \%$ or more of annual prescription fills dispensed by a long-term care pharmacy. Frequencies of drug receipt were compared between community-dwelling and LTC beneficiaries using the chi-square test; $p$ value $<0.05$ was considered significant. All three comparisons have $p$ value $<0.001$.

This population may warrant an explicit effort to examine and quantify the risk-benefit tradeoff associated with the use of FADs, especially concurrent FADs.

This study is limited by the use of administrative data; prescription fills are a proxy for exposure. We cannot assess clinical appropriateness of prescribing.

Future studies should quantify fracture risk associated with observed drug receipt in this population and relative risk associated with distinct combinations. Future quality metrics could incorporate overlapping adverse drug effects or total FAD burden. ${ }^{1}$
Rebecca T. Emeny, PhD, MPH ${ }^{1}$

John A. Batsis, $M D^{1,2,3}$

Nancy E. Morden, MD, MPH ${ }^{1}$

${ }^{1}$ The Dartmouth Institute for Health Policy \& Clinical Practice, The Geisel School of Medicine at

Dartmouth ,

Hanover, NH, USA

${ }^{2}$ Section of General Internal Medicine, Dartmouth-

Hitchcock Medical Center,

Lebanon, NH, USA 
Table 1 Ten Most Common Single Fracture-Associated Drug and Concurrent Two-Way Fracture-Associated Drug Combinations Among Medicare Beneficiaries in Long-term Care Compared with the Community-Dwelling Medicare Population

\begin{tabular}{|c|c|c|c|c|}
\hline & \multicolumn{2}{|c|}{$\begin{array}{l}\text { Medicare beneficiaries in long-term care } \\
(n=422,111)\end{array}$} & \multicolumn{2}{|c|}{$\begin{array}{l}\text { Community-dwelling Medicare } \\
\text { population }(n=4,868,813)\end{array}$} \\
\hline & Person years & $\%$ Total person years & Person years & $\%$ Total person years \\
\hline Total observed time & $1,803,544$ & & $20,759,698$ & \\
\hline Single fracture-associated drugs receipt & 371,838 & 20.62 & $5,281,454$ & 25.44 \\
\hline Concurrent two-way fracture-associated drug receipt & 272,620 & 15.12 & $2,371,101$ & 11.42 \\
\hline \multicolumn{5}{|l|}{ Receipt of specific individual fracture-associated drugs } \\
\hline Thiazide diuretics & 75,155 & 4.17 & $2,105,826$ & 10.14 \\
\hline SSRIs & 56,425 & 3.13 & 436,347 & 2.10 \\
\hline Loop diuretics & 48,960 & 2.71 & 420,953 & 2.03 \\
\hline PPIs & 43,882 & 2.43 & 787,238 & 3.79 \\
\hline 2nd-generation antispychotics & 29,017 & 1.61 & 27,926 & 0.13 \\
\hline Opioids & 19,747 & 1.09 & 238,252 & 1.15 \\
\hline Anticonvulsants & 18,353 & 1.02 & 177,986 & 0.86 \\
\hline $\mathrm{H} 2$ receptor antagonists & 12,917 & 0.72 & 111,449 & 0.54 \\
\hline Nitrates & 10,114 & 0.56 & 120,585 & 0.58 \\
\hline Antiparkinson agents & 10,101 & 0.56 & 65,484 & 0.32 \\
\hline \multicolumn{5}{|l|}{ Receipt of specific concurrent fracture-associated drugs } \\
\hline 2nd generation antispychotics \& SSRIs & 17,098 & 0.95 & 16,085 & 0.08 \\
\hline PPIs \& SSRIs & 15,634 & 0.87 & 112,261 & 0.54 \\
\hline SSRIs \& loop diuretics & 13,676 & 0.76 & 39,485 & 0.19 \\
\hline SSRIs \& thiazide diuretics & 13,320 & 0.74 & 157,652 & 0.76 \\
\hline PPIs \& loop diuretics & 12,834 & 0.71 & 88,497 & 0.43 \\
\hline PPIs \& thiazide diuretics & 11,537 & 0.64 & 320,210 & 1.54 \\
\hline Anticonvulsants \& SSRIs & 6482 & 0.36 & 29,025 & 0.14 \\
\hline 2nd-generation antispychotics \& loop diuretics & 5657 & 0.31 & 2772 & 0.01 \\
\hline SSRIs \& opioids & 5635 & 0.31 & 33,132 & 0.16 \\
\hline PPIs \& 2nd-generation antispychotics & 5596 & 0.31 & 4740 & 0.02 \\
\hline
\end{tabular}

For fracture-associated drugs, status of receipt of 21 single drugs and concurrent two-way drug combinations is classified for each person, for each day of observation. Beneficiaries classified as living in long-term care (LTC) are those with 50\% or more annual prescription fills dispensed by a LTC pharmacy. SSRIs selective serotonin reuptake inhibitors and selective norepinephrine reuptake inhibitors, PPIs proton pump inhibitors. Chi-square test was used to compare frequencies of drug receipt between LTC and community-dwelling Medicare beneficiaries; all comparisons are significant with $p$ value $<0.001$

${ }^{3}$ Dartmouth Centers for Health and Aging, Dartmouth College, Hanover, NH, USA

Corresponding Author: Rebecca T. Emeny, PhD, MPH; The Dartmouth Institute for Health Policy \& Clinical Practice, The Geisel School of Medicine at Dartmouth Hanover, NH, USA (e-mail: rebecca.t.emeny@dartmouth.edu).

Funding Information RTE and NEM were supported by The Dartmouth Clinical and Translational Science Institute, under award number UL1TRO01086 from the National Center for Advancing Translational Sciences (NCATS) of the National Institutes of Health (NIH) and by National Institute on Aging: PO1 AG019783, Skinner (PI): Causes and Consequences of Healthcare Efficiency.

$J B$ was funded in part by the National Institute on Aging of the National Institutes of Health under Award Number K23AG051681.

\section{Compliance with Ethical Standards:}

Conflict of Interest: The authors declare that they do not have a conflict of interest.
Disclaimer: The views expressed in this article are those of the authors and no endorsement by the NIA or the NIH is intended or should be inferred.

\section{REFERENCES}

1. Emeny RT, Chang CH, Skinner J, et al. Association of Receiving Multiple, Concurrent Fracture-Associated Drugs With Hip Fracture Risk. JAMA Netw Open. 2019;2(11):e1915348.

2. Berry SD, Lee Y, Zullo AR, Kiel DP, Dosa D, Mor V. Incidence of Hip Fracture in U.S. Nursing Homes. $J$ Gerontol A Biol Sci Med Sci. 2016;71(9):1230-1234.

3. Harris-Kojetin L, Sengupta M, Park-Lee E, Valverde R, Caffrey C, Rome $\mathbf{V}$, Lendon $\mathbf{J}$. Long-term care providers and services users in the United States: Data from the National Study of Long-Term Care Providers, 20132014. Vital Health Stat 3. 2016;3(38).

4. By the American Geriatrics Society Beers Criteria Update Expert P. American Geriatrics Society 2019 Updated AGS Beers Criteria(R) for Potentially Inappropriate Medication Use in Older Adults. J Am Geriatr Soc. 2019:67(4):674-694.

Publisher's Note: Springer Nature remains neutral with regard to jurisdictional claims in published maps and institutional affiliations. 